\title{
Research Progress on Classical Traditional Chinese Medicine Jieyu Pills in the Treatment of Depression
}

This article was published in the following Dove Press journal: Neuropsychiatric Disease and Treatment

\section{Yuan Wang (1D \\ Miao Peng (D)}

Department of Psychiatry, Shengjing Hospital of China Medical University, Shenyang, Liaoning, People's Republic of China
Correspondence: Miao Peng Department of Psychiatry, Shengjing Hospital of China Medical University, Heping District, Shenyang II 0004, Liaoning, People's Republic of China $\mathrm{Tel} / \mathrm{Fax}+8624$ I89402527I0

Email pengmiao_cmu@yeah.net

\begin{abstract}
Depression is a common clinical psychological disease, which is called "yu zheng" in traditional Chinese medicine (TCM). TCM has a long history in the treatment of depression (yu zheng), which has unique advantages. Jieyu pill (JYP), a classical TCM formula, has been widely used for treating depression because of its clear clinical efficacy, low side effects, and high compliance. In this review, we systematically introduce recent clinical and animal experimental studies on JYP and depression, and review the pharmacological mechanism and active ingredients of JYP, as well as its clinical application in depression therapy. This systematic review provides a deep understanding of TCM prescriptions, pharmacological mechanisms, and disease-medicine interactions, and lays the foundation for developing new treatments for depression.
\end{abstract}

Keywords: Jieyu pill, depression disorder, yu zheng, pharmacology, active ingredient

\section{Introduction}

Depression disorder is one of the most common psychological diseases in a clinical context. According to the International Classification of Diseases (ICD-11) published by the World Health Organization (WHO), depression is characterized by a lack of pleasure, accompanied by impairments in cognitive, behavioral, and autonomic functions, which seriously affect the patient's social function. ${ }^{1}$ According to statistics released by the WHO in 2017, there are 322 million depressive patients globally, with an overall prevalence of $4.4 \%$. The prevalence rates in women and men are $5.1 \%$ and $3.6 \%$, respectively. Depressive disorder is a major cause of disability globally and a major factor in the global disease burden. ${ }^{2}$ However, less than half of patients globally receive anti-depression therapy, and there are still great difficulties and challenges regarding the diagnosis and treatment of depression. ${ }^{3}$

Traditional Chinese medicine (TCM) is an experience-based medicine that has been developed in China over thousands of years. After repeated clinical practice, a TCM formula is established, which is typically composed of multiple drugs and can act on multiple organs and targets. This multichannel treatment method is compatible with the multifactorial pathological mechanism of depression. A variety of TCM formulas have been used clinically in the treatment of depressive disorders. Meta-analysis has shown that the treatment of depressive disorders with Chinese medicine is superior to a placebo in terms of improving symptoms. Its effectiveness is similar to that of Western medicine antidepressants, but with a lower incidence of adverse events. ${ }^{4}$ 


\section{Depression and Yu Zheng}

In TCM, it is believed that a depressed mood is caused by emotional trauma, and is classified as "yu zheng." In the "Jingyue Quanshu," people believed that all diseases originated from five qi stasis, which emphasized the importance of "yu zheng" in human diseases. Previous studies found that the four common symptoms of depression disorder are 1) liver qi stagnation, 2) liver stagnation and spleen deficiency, 3) heart and spleen deficiency, liver stagnation and qi stagnation, and 4) liver and kidney yin deficiency, with liver qi stagnation being the most common. ${ }^{5}$ In TCM, it is believed that "yu zheng" is mainly caused by stagnation of liver qi. The disease is located in the liver and closely associated with the spleen, kidney, and heart. Therefore, the treatment should be based on the liver, which should relieve the depression of the liver and ventilate the qi and blood, and make the functions of the viscera, the whole body, and the organs stable and orderly, so that the emotions can be smoothed.

JYP is a pure Chinese medicine formula refined by modern science and technology. As a commercial drug, JYP is widely used in clinic. It was developed on the basis of ancient formulas, namely, Xiaoyao decoction and Ganmai Dazao decoction, and is composed of Paeoniae Radix Alba, Radix Bupleuri, Angelicae Sinensis Radix, Curcumae Radix, Poria cocos (Schw.) Wolf., and Jujubae Fructus. Paeoniae Radix Alba nourishes the blood and soothes the liver, while Radix Bupleuri soothes the liver and regulates qi. Curcumae Radix not only effects on the qi but also on the blood, which can relieve qi stasis and cool the clearing heart. It is more effective at nourishing the blood and promoting blood circulation in combination with Angelicae Sinensis Radix. Four drugs constitute the classic prescription Xiaoyao decoction, which is used to treat fullness of chest hypochondrium, and depression. Poria cocos (Schw.) Wolf. and Jujubae Fructus have the effects of nourishing the heart, relieving depression and removing anxiety, and treating palpitations, insomnia, and dreaminess. JYP is a brown to tan water pill, slightly bitter, weighing 1 gram per 15 pills. When the six drugs are used together, JYP has the greatest therapeutic effect on "yu zheng" (depression disorder) in biomedicine.

\section{Pharmacological Studies on the Active Ingredients of the Six Chinese Medicines Comprising JYP}

\section{Paeoniae Radix Alba (PRA)}

PRA is the dried root of Paeonia tacti lora Pall., it has the functions of nourishing the blood and regulating menstruation, condensing the yin and antiperspirant, softening the liver and relieving pain, and calming the liver and yang. It is commonly used for blood deficiency, irregular menstruation, spontaneous sweating, night sweats, flank pain, abdominal pain, limb pain, headache, and dizziness. ${ }^{6,7}$ Previous studies showed that PRA could be used for anti-inflammatory effects, ${ }^{8}$ immune regulation, ${ }^{9}$ treatment of depression ${ }^{10,11}$ and anemia, ${ }^{12}$ promotion of peripheral nerve regeneration, ${ }^{13}$ and treatment of viral infections. ${ }^{14}$

Modern studies of pharmacological mechanisms have found that the main active components of PRA include paeoniflorin, paeonol, paeonin, benzoylpaeoniflorin, albiflorin, oxypaeoniflorin, paeoniflorigenone, benzoic acid, $\beta$-sitosterol, and gal-lotannin. Paeoniflorin is the main pharmacological component of PRA. It has been reported to have neuroprotective effects in a transgenic mouse model of Alzheimer's disease (AD), ${ }^{15}$ can influence the prognosis of chronic heart failure (CHF), ${ }^{16}$ ameliorate antipsychotic drug-induced side effects, ${ }^{17}$ attenuate early brain injury through reducing oxidative stress and neuronal apoptosis after subarachnoid hemorrhage in rats, ${ }^{18,19}$ and exert antitumor effects, ${ }^{20}$ protective effects on acute cerebral infarction, ${ }^{21}$ and anti-inflammatory and immunoregulatory effects, ${ }^{22}$ as well as promote neurogenesis in the hippocampal dentate gyrus of depressive-like rats. $^{23}$

\section{Radix Bupleuri (RB)}

$\mathrm{RB}$ is an umbelliferous plant, which is mostly dug up in spring and fall. It is commonly used as an antidote. It is bitter and slightly cold, belonging to the channels of liver and gall bladder. RB has the effects of reconciling superficies and interior, complementing qi, and soothing the liver. It is used to treat liver qi stagnation, chest and rib pain, anal prolapse, uterine prolapse, and irregular menstruation. In recent years, research on the application of RB has mainly focused on its use against depression, ${ }^{24}$ for inhibiting cancer growth and metastasis, ${ }^{25}$ anti-hepatitis B virus (HBV) effects, ${ }^{26}$ antioxidative, anti-inflammatory, and hepatoprotective effects, ${ }^{27}$ antimicrobial, antiviral, hepatoprotective, neuroprotective, and immunomodulatory effects, ${ }^{28,29}$ antiepileptic effect, ${ }^{30}$ and its use for treating $\mathrm{AD} .{ }^{31}$

Although there is an extensive range of clinical applications of RB, recent research has mainly focused on its antidepressant effect. Through the study of the pharmacological mechanism of RB, it has been found that its main active ingredients include saikosaponin, sterols, volatile oils (chaihuol, eugenol), fatty acids (oleic acid, linoleic acid, palmitic 
acid, stearic acid), and polysaccharides. Among these, the main ingredient is saikosaponin. Saikosaponin has the following positive effects: antitumor ${ }^{32}$ and antiviral activities, ${ }^{33}$ regulation of triglycerides, ${ }^{34}$ antidepression effects, ${ }^{35}$ the prevention and treatment of atherosclerosis, improvement of memory deficits, ${ }^{36}$ antiepileptic effects, ${ }^{37}$ the suppression of inflammatory responses ${ }^{38}$ and reduce depressive, anxietylike behavior in chronic corticosterine-treated mice [10.1186/ s12906-018-2186-9].

\section{Angelicae Sinensis Radix (ASR)}

ASR is the dried root of Umbelliferae Angelica sinensis (Oliv) Diels. In terms of its properties, it is sweet, spicy, and warm, belonging to the channels of liver, heart, and spleen. The main functions of ASR include nourishing the blood, promoting blood circulation, regulating menstruation and analgesia, moisturizing and smoothing the intestines, and relieving abdominal pain due to cold deficiency and numb skin. Recent studies have also found that ASR can be used to treat idiopathic pulmonary fibrosis, ${ }^{39}$ depression, ${ }^{40}$ and multiple sclerosis. ${ }^{41}$ It also has the effects of ameliorating memory deficiency ${ }^{42}$ and promoting axon regeneration after ischemic brain injury, ${ }^{43}$ as well as exerting antiinflammatory ${ }^{44}$ and neuroprotective effects, ${ }^{45}$ and promoting synaptic plasticity during cognitive recovery. ${ }^{46}$

Studies of its pharmacological mechanisms have confirmed that ASR has many active ingredients, including butylidenephthalide, $\beta$-pinene, $\alpha$-pinene, camphene, p-cymene, $\beta$-phellandrene, myrcene, allo-ocimene, 6-n-butyl-cycloheptadiene-1, acetophenone, $\beta$-bisabolene, isoacroraene, acoradiene, chamigrene, $\alpha$-cedrene, and bergapten. Among these, butylidenephthalide is the most studied and widely used. Studies have shown that butylidenephthalide can prolong the lifespan and attenuate motor neuron loss in a superoxide dismutase 1 (SOD1) mouse model of amyotrophic lateral sclerosis (ALS), ${ }^{47}$ act in adjuvant therapy of malignant human gliomas, ${ }^{48}$ rehabilitate the neurovascular unit in rats after photothrombotic stroke, ${ }^{49}$ exhibit therapeutic effects in a mouse model of Parkinson's disease (PD) ${ }^{50}$ protect against neurotoxicity in spinocerebellar ataxia type $3,{ }^{51}$ attenuate AD-like cytopathy in Down syndrome, ${ }^{52}$ and prevent inflammation-mediated neurotoxicity. ${ }^{53}$

\section{Curcumae Radix (CR)}

$\mathrm{CR}$ is the dried root of Curcuma rcenyujin $Y, H$. Chenet

C. Ling. It is usually dug up after the stems and leaves wither in winter. In terms of the properties of $\mathrm{CR}$, it is spicy, bitter, and cold, belonging to the channels of liver, heart, and lungs. The effects of CR include: promoting blood circulation and relieving pain, relieving qi stagnation, clearing the heart, and cooling the blood. It can be used for chest obstruction with pain, epilepsy, amenorrhea, and oligomenorrhea. In addition, CR has anti-inflammatory and antinociceptive, ${ }^{54}$ antiepilepsy, ${ }^{55}$ antitumor, ${ }^{56}$ and antidepression effects, ${ }^{57}$ while also countering autoimmune liver disease $^{58}$ and atherosclerosis, exerting cardioprotective effects, ${ }^{59}$ and assisting in the treatment of AD. ${ }^{60}$

It has been reported that $\mathrm{CR}$ root contains curcumin, aromatic curcumin, gingerene, cressene, cypressene, eucalyptol, borneol, camphor, and curcumin. Among these, research on curcumin has been most intensively performed, and curcumin is currently considered to have the highest medicinal value among these components. Curcumin has been used for the adjuvant treatment of depression, ${ }^{61}$ shown to exert beneficial effects on depression and anxiety in diabetic patients, ${ }^{62}$ and to improve unpredictable chronic mild stress-induced depression and memory deficits. ${ }^{63}$ In addition, curcumin was reported to prevent or delay the onset of various neurological diseases, such as AD, PD, Huntington's disease (HD), multiple sclerosis, and ALS. ${ }^{64}$

\section{Poria cocos (Schw.) Wolf (PCW)}

$\mathrm{PCW}$ is the dried sclerotia of the fungus Poria cocos (Schw.) Wolf in the Polyporaceae family. In terms of the properties of PCW, it is sweet and light, belonging to the heart, lung, spleen, and kidney channels. Its pharmacological effects include diuresis and dampness, strengthening the spleen, and calming the heart. It is mainly used to treat edema and oliguria, sputum drinking and dizziness, spleen deficiency, restlessness, convulsions, and insomnia. Clinically, PCW is mainly used for treating renal fibrosis, ${ }^{65}$ osteoporosis, ${ }^{66}$ malignant tumors, ${ }^{67}$ cerebral ischemia/reperfusion injury, ${ }^{68}$ depression, ${ }^{69}$ fatty liver, ${ }^{70}$ diabetes, ${ }^{71}$ and AD. ${ }^{72}$

PC is abundantly available, and its pharmacological effects are remarkable. So far, the main chemical components that have been separated from PC include poria glycans, pachymic acid, as well as sterols, choline, adenine, lecithin, and histidine acid. The pharmacological effects of pachymic acid include antitumor activity, ${ }^{73}$ improving learning and memory impairment, ${ }^{74}$ protecting against cerebral ischemia/reperfusion injury, ${ }^{68}$ ameliorating renal failure, ${ }^{75}$ improving survival and attenuating acute lung injury, ${ }^{76}$ improving sleep, ${ }^{77}$ and exerting anti-inflammatory ${ }^{78}$ and 
antihyperglycemic effects. ${ }^{79}$ In addition, pachymic acid has clear antidepressant and antianxiety effects. ${ }^{80,81}$

\section{Jujubae Fructus (JF)}

JF belongs to the genus Zizyphus of the Rhamniaceae family. It is sweet in taste and warm, belonging to the spleen and stomach channels. JF has the functions of supplementing qi, nourishing the blood, calming the mind, and relieving drug resistance. It exerts optimal effects for treating allergic purpura, anemia, hypertension, acute and chronic hepatitis, and cirrhosis, as well as preventing blood transfusion reactions. JF contains triterpenoid compounds and cyclic adenosine phosphate, having strong anticancer and antifatigue effects. JF also has the effect of reducing damage due to toxic substances in the liver, while the flavonoids in JF have sedative and bloodpressure-lowering effects.

JF mainly contains organic acids, triterpene glycosides, alkaloids, and flavonoids. These organic acids include betulinic acid and oleanolic acid; the triterpene glycosides include maslinic acid-3-O-trans-p-coumarinyl ester and maslinic acid-3-O-cis-p-coumarinyl ester; the alkaloids include stepharine and n-nornuciferine; and the flavonoids include 6,8-di-C-glucosyl-2(S)-naringenin, 6.8-di$\mathrm{C}$-glucosyl-2(R)-naringenin, and rutin. Betulinic acid is one of the main effective ingredients of JF. Studies have shown that it has therapeutic effects on various diseases, such as influenza virus infection, ${ }^{82}$ glioblastoma, ${ }^{83}$ ischemic stroke, ${ }^{84}$ and a variety of malignant tumors. ${ }^{85,86}$ In addition, betulinic acid has unique effects on improving $\mathrm{AD}$ and combating anxiety/depression. ${ }^{87,88}$

\section{The Clinical Application of JYP in the Treatment of Depression}

In recent years, with the deepening of people's understanding of TCM, JYP and its modified versions have been widely used in the treatment of depression and related psychological disorders. JYP was shown to be effective in treating depression, and studies have reported that the rate at which it was effective was $78.8 \%$, which is equivalent to the efficacy of treatment with the Western medicine maprotiline. JYP can also significantly improve patients' Hamilton Depression Rating Scale (HAMD) score, Self-rating Scale for Depression (SDS) score, Selfrating Scale for Anxiety (SAS) score, and Clinical Global Impression (CGI) score. In addition, Shen et al showed through randomized controlled trials that JYP is as effective as maprotiline in the treatment of depression, but has fewer side effects. ${ }^{89}$ Adding JYP to Western medicine in the treatment of depression could thus achieve good results. Liu et al collected and followed up 32 patients with refractory depression (RD) in the form of yin deficiency inner heat syndrome (YDIHS), treated with JYP and paroxetine, and evaluated their HAMD and Hamilton anxiety scale scores at the second, fourth, and eighth weeks. They found that JYP combined with paroxetine was superior to the use of paroxetine alone. ${ }^{90}$ Moreover, in a comparative study of the efficacy of JYP combined with sertraline versus sertraline alone in the treatment of depression, $\mathrm{Qu}$ et al found that the improvement of depressive symptoms was more pronounced in patients treated with JYP. Moreover, the levels of the inflammatory factors interleukin-2 (IL-2), IL-6, tumor necrosis factor- $\alpha$ (TNF- $\alpha$ ), and IL-1 $\beta$ were significantly reduced, while 5-serotonin (5-HT), norepinephrine (NE), and dopamine (DA) levels were significantly increased in the serum of patients receiving JYP treatment, suggesting that JYP combined with sertraline could effectively improve the depressive state of patients and promote the secretion of monoamine neurotransmitters. Furthermore, Chen et al performed a study including 116 patients with depression, to whom JYP combined with Shugan Jieyu capsule therapy was applied. Through a 6-week followup study of these patients, it was found that the depressive symptoms were significantly alleviated in patients receiving JYP treatment, which did not increase the incidence of adverse effects. The study also suggested that JYP could improve the quality of sleep of patients with depression. ${ }^{91}$ JYP combined with citalopram can improve the symptoms of patients with $\mathrm{RD}$, and can significantly increase the serum 5-HT, NE, and brain-derived neurotrophic factor (BDNF) levels in patients, suggesting that the mechanism of JYP to improve depressive symptoms may be related to the regulation of monoamine transmitter levels and the activation of BDNF expression. In addition to improving depressive symptoms, JYP could also improve stroke symptoms and the ability to perform daily living activities among patients with post-stroke depression, so JYP is also recommended for such patients. ${ }^{92}$

In summary, these clinical studies have shown that JYP alone or combined with other TCM or Western medicines is effective in treating depression, and has fewer side effects than conventional drugs. The mechanism by which JYP acts in the treatment of depression is relatively complex. Clinical studies performed to date have found 
that it may rely on increasing 5-HT, NE, and DA levels. From the dialectical perspective of TCM, JYP mainly treats depressive patients by improving liver qi stagnation syndrome, liver stagnation, and spleen deficiency syndrome. Next, we review the possible mechanisms by which JYP acts in treating depression.

\section{The Pharmacological Mechanism of JYP in the Treatment of Depression}

With the increasing clinical application of JYP, research on its pharmacological mechanism has also received more and more attention. Wang et al suggested that JYP has an ameliorative effect on post-stroke depression in rats via modulating BNDF levels, cannabinoid receptors, and corticotrophin-releasing factor in ventral tegmental area-nucleus accumbens tissue. ${ }^{93}$ Paeoniflorin, an active ingredient of PRA, can improve the depression-like behavior caused by chronic stress. This antidepressant effect may occur by regulating the functional activity of the hypothalamic-pituitary-adrenal (HPA) axis and increasing BDNF levels in the brain. ${ }^{94}$ It was reported the paeoniflorin could also ameliorate depressive-like behavior by decreasing the degree of neuronal damage in the hippocampus of model rats. In addition, paeoniflorin was reported to exert a neuroprotective effect by modulating the ERK-CREB signaling pathway in rats with chronic unpredictable mild stress-induced hippocampal damage. ${ }^{95}$ Pharmacodynamic tests showed that paeoniflorin had significant antidepressant activity through its metabolism into benzoic acid via gut microbiota enzymes, which might exert antidepressant effects by passing through the blood-brain barrier into the brain. ${ }^{96}$ Saikosaponin could also effectively improve lipopolysaccharide-induced inflammation-related depressive-like behaviors by inhibiting microglia activation and neuroinflammation. ${ }^{35}$ Moreover, Chao et al suggested that saikosaponin could ameliorate depression-like behaviors in rats by downregulating nuclear factor- $\mathrm{kB}$ (NF$\kappa \mathrm{B})$ and miR-155, and upregulating fibroblast growth factor 2 (FGF2). ${ }^{97}$ The results of Gong et al demonstrated that ASR could significantly improve chronic unpredictable mild stress-induced depressive symptoms, hematological anomalies, and hypoxia symptoms by modulating energy metabolism and inhibiting the expression of pyruvate dehydrogenase lipoamide kinase isozyme 1 and lactate dehydrogenase $\mathrm{A}^{40}$ The butylidenephthalide in ASR could regulate the nuclear factor E2 related factor
2 and NF- $\mathrm{BB}$ pathway, produce anti-inflammatory effects, and improve lipopolysaccharide-induced depression-like behavior. ${ }^{98}$ Curcumin is widely used as an antiinflammatory agent and has the potential to combat depression. It exerts anti-depression effects by inhibiting P2X7R expression, which then deactivates the $\mathrm{Ca}^{2+}$ channel-mediated inflammatory response associated with the exacerbation of depression. ${ }^{99}$ Naqvi et al reported that curcumin supplementation significantly attenuated unpredictable chronic mild stress-induced depression, decreased the load of unpredictable chronic mild stress propagated oxidative stress by improving antioxidant enzyme activities. ${ }^{63}$ Curcumin also attenuated IL-1 $\beta$ induced neuronal apoptosis, which may be related to depression-like behavior in stressed rats. ${ }^{100}$ Moreover, curcumin effectively decreased the levels of proinflammatory cytokines (TNF- $\alpha$, IL-1 $\beta$, and IL-6) and suppressed NF- $\mathrm{kB}$ activation, while also inhibiting stressinduced P2X7R/NLRP3 inflammatory axis activation, along with reducing the transformation of pro-IL-1 $\beta$ to mature IL-1 $\beta$, thereby relieving the depression-like state through inhibiting the NLRP3 inflammatory pathway. ${ }^{101}$

This review of previous studies clearly shows that various active ingredients in JYP can produce different therapeutic effects through different targets in depression therapy. Different ingredients in JYP may increase the levels of monoamine neurotransmitters in the brain, adjust the HPA axis, and produce anti-inflammatory effects and other regulatory effects on energy and substance metabolism pathways to synergistically exert antidepressant effects, suggesting the broad potential of JYP in the treatment of depression.

\section{The Active Compounds of JYP in the Treatment of Depression}

In TCM, the treatment of depression is usually based on an overall analysis of the illness and the patient's condition, indepth analysis of the etiology and pathogenesis of depressive disorder, correction and selection of prescriptions during repeated consultations, and ultimately relieving the depressive symptoms. The antidepressant effect of JYP is a synergistic effect of multiple effective ingredients, with the therapeutic effect occurring through the synergistic activities of multiple drugs. This therapeutic effect thus involves the characteristics of multiple ingredients, multiple targets, and multiple pathways. As such, it is difficult to study the 
<smiles>CC12CC3(O)OC(C1O)C(COC(=O)c1ccccc1)(C2)C31CCC1(O)OC1OC(CO)C(O)C(O)C1O</smiles>

Paeoniflorin<smiles>CCCC=C1OC(=O)c2ccccc21</smiles>

Butylidenephthalide

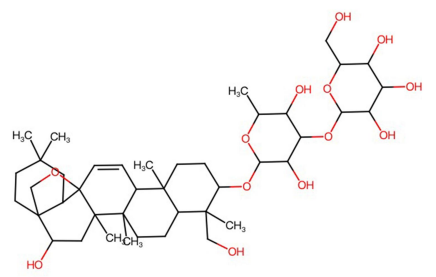

Saikosaponin

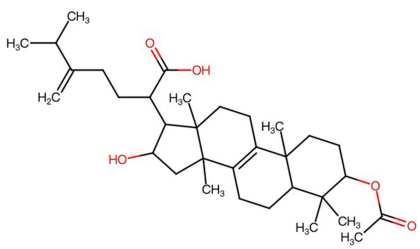

Pachymic acid

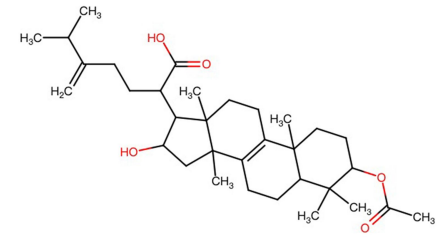

Betulinic acid

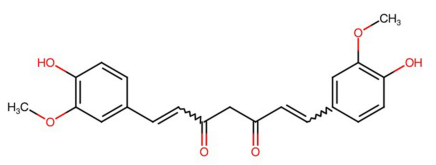

Curcumin

Figure I The 2D structures of effective biomarkers in JYP (JYP, Jieyu pill).

pharmacological mechanism of TCM formula comprehensively by traditional experimental methods.

Network pharmacology is a new field of study based on the theory of systems biology, which analyzes networks of biological systems and selects specific signal nodes for the design of multiple targeted drugs. Basing on the parameters of distribution, absorption, metabolism and excretion, we first screened the active ingredients of JYP by

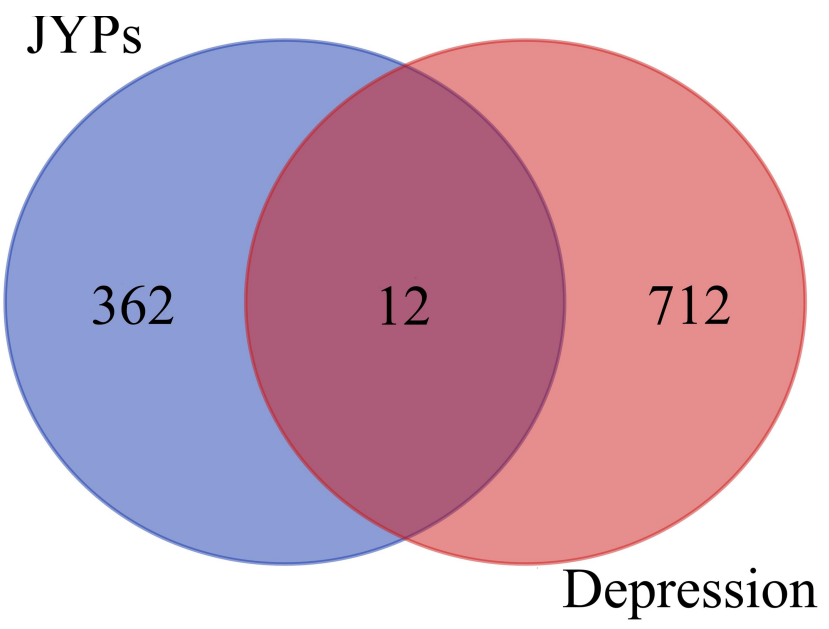

Figure 2 A total of 12 targets of both JYP and depression were identified. Abbreviations: JYP, Jieyu pill; TCM, traditional Chinese medicine. searching the pharmacological database of the traditional Chinese medicine system platforms (TCMSP) (http:// www.tcmspw.com/). A total of six active ingredients in JYP were identified, including paeoniflorin, saikosaponin,

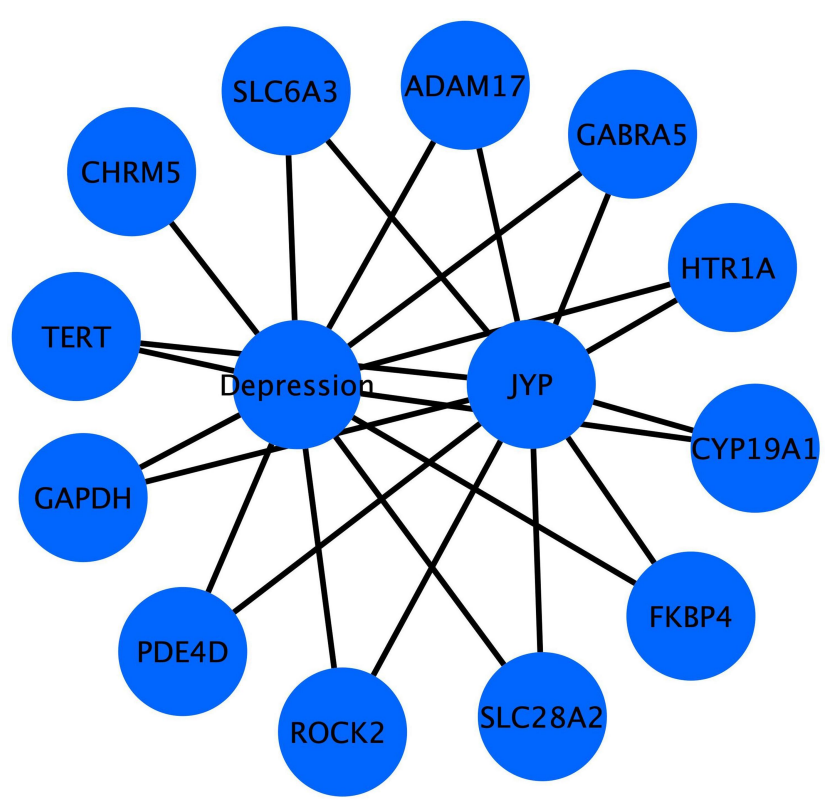

Figure $3 \mathrm{~A}$ total of 12 targets were used to establish the regulatory network in Cytoscape.

Abbreviation: JYP, Jieyu pill. 

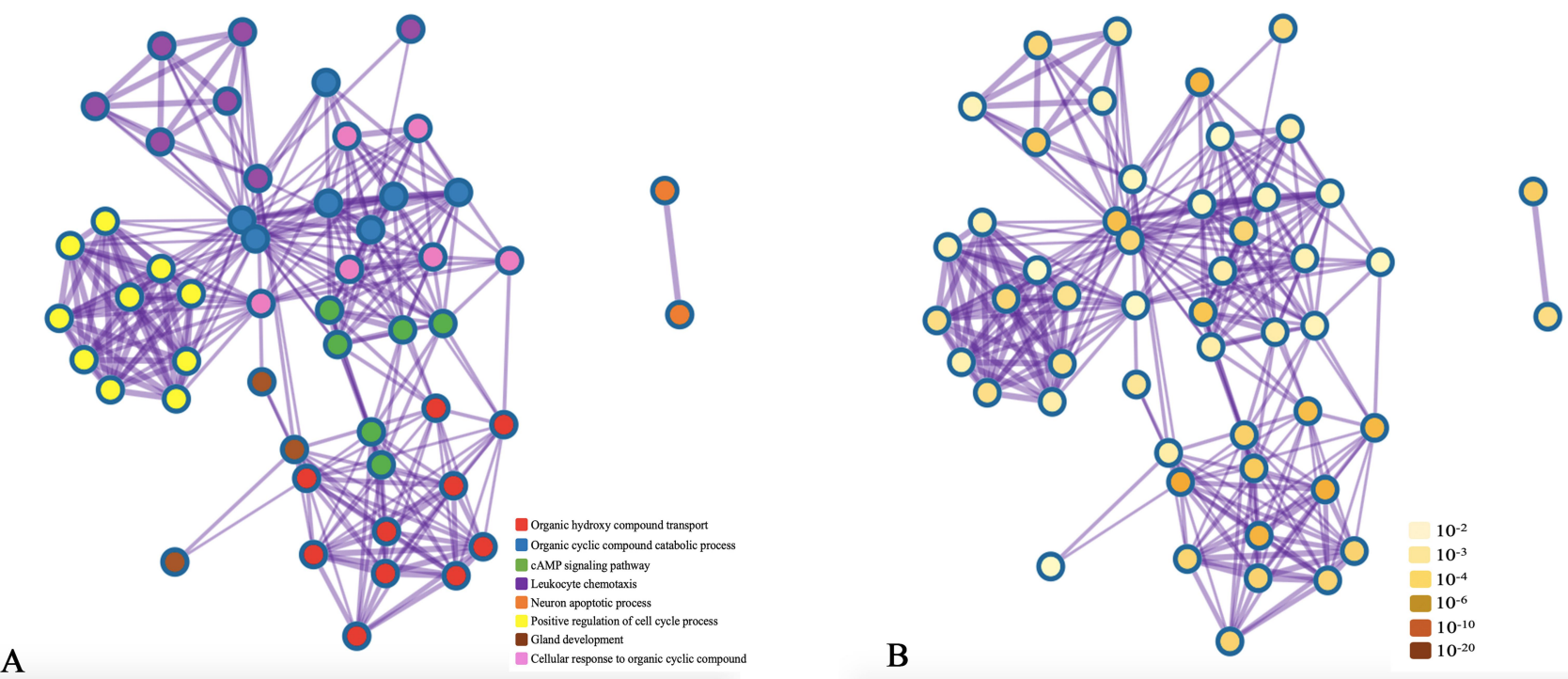

Figure 4 Network of enriched terms: (A) colored by cluster ID, where nodes that share the same cluster ID are typically close to each other; (B) colored by $p$-value, where terms containing more genes tend to have a more significant $p$-value.

butylidenephthalide, curcumin, pachymic acid and betulinic acid. The 2-D structure and the Canonical SMILES of eight effective biomarkers in JZG were obtained from PubChem (https://pubchem.ncbi.nlm.nih.gov/) (Figure 1). The Swiss Target Prediction database (http://www.swisstar getprediction.ch/) and Lab of Systems Pharmacology (http://www.tcmspw.com/) were used to screen the targets of the active biomarkers in JYP. The targets of depression were obtained from the Therapeutic Targets database (http://bidd.nus.edu.sg/group/cjttd/) and the OMIM database (http://omim.org/).

Ultimately, 362 target genes of JYP and 712 target genes of depression were identified; as a result, 12 collective targets of depression and JYP were obtained (Figure 2). Metascape (http://metascape.org) provides automated meta-analysis tools to understand common and unique approaches in a set of orthogonal target discovery studies. It also supports BioGrid-based protein-protein interaction (PPI) analysis and interactive visualization of Gene
Ontology (GO) networks. ${ }^{102}$ The network of enriched terms was analyzed by Metascape (Figure 3) and the PPI was constructed by Cytoscape (Figure 4). The top eight clusters with representative enriched terms of JYP's actions on depression are shown in Table 1. We speculate that JYP may treat depression via these enriched pathways.

\section{Conclusion and Prospects}

Depression is a psychological disorder with a high prevalence rate, for which drug treatment is currently the main therapeutic method. Drug selection should be based on a variety of factors, including: treatment response to previous drugs; drug safety, tolerability, and adverse reactions; concomitant mental or physical illnesses; and pharmacological characteristics of the drugs. Western medicine antidepressants have significant therapeutic effects, but their safety, tolerance, and compliance are relatively poor. JYP was innovatively developed on the basis of a TCM prescription; it is mainly used for the

Table I Top eight Clusters with Their Representative Enriched Terms of JYPs on the Depression

\begin{tabular}{|l|l|l|l|l|l|}
\hline GO & Category & Description & Count & $\%$ & Log I0 (P) \\
\hline GO:0015850 & GO Biological Processes & Organic hydroxy compound transport & 4 & 33.33 & -5.19 \\
GO:I,901,36I & GO Biological Processes & Organic cyclic compound catabolic process & 5 & 41.67 & -4.68 \\
GO:0030595 & GO Biological Processes & Leukocyte chemotaxis & 3 & 25.0 & -3.79 \\
GO:005I402 & GO Biological Processes & Neuron apoptotic process & 3 & 25.0 & -3.68 \\
GO:0090068 & Canonical Pathways & Positive regulation of cell cycle process & 3 & 25.0 & -3.40 \\
GO:0048732 & GO Biological Processes & Gland development & 3 & 25.0 & -2.93 \\
GO:007I407 & GO Biological Processes & Cellular response to organic cyclic compound & 3 & 25.0 & -2.67 \\
\hline
\end{tabular}


treatment of depression, upset, insomnia, and dreaminess caused by the stagnation of liver qi and restlessness. In clinical practice, it has been found that JYP has good clinical efficacy in the treatment of depressive disorders and accompanying symptoms. In combination with Western medicine antidepressants, it not only has a synergistic effect, but also reduces adverse reactions and has high compliance. Here, the effects of JYP on depression have been systematically reviewed from the perspectives of composition, animal experiments, pharmacological actions, effective components, and clinical applications. Through these studies, we hope to provide a comprehensive and profound understanding of the mechanism by which JYP acts on depression, and scientific guidance for JYP's wider clinical application.

\section{Author Contributions}

All authors made substantial contributions to conception and design, acquisition of data, or analysis and interpretation of data; took part in drafting the article or revising it critically for important intellectual content; agreed to submit to the current journal; gave final approval of the version to be published; and agreed to be accountable for all aspects of the work.

\section{Funding}

There is no funding to report.

\section{Disclosure}

The authors declare that they have no competing interests for this work.

\section{References}

1. Chute CG. The rendering of human phenotype and rare diseases in ICD-11. J Inherit Metab Dis. 2018;41(3):563-569. doi:10.1007/ s10545-018-0172-5

2. Spencer L J, Abate D, Abate KH, et al. Global, regional, and national incidence, prevalence, and years lived with disability for 354 diseases and injuries for 195 countries and territories, 1990-2017: a systematic analysis for the global burden of disease study 2017. Lancet. 2018;392 (10159):1789-1858.

3. Sowa-Kućma M, Pańczyszyn-Trzewik P, Misztak P, et al. Vortioxetine: a review of the pharmacology and clinical profile of the novel antidepressant. Pharmacol Rep. 2017;69(4):595-601.

4. Yeung WF, Chung KF, Ng KY, Yu YM, Ziea ET, Ng BF. A systematic review on the efficacy, safety and types of Chinese herbal medicine for depression. J Psychiatr Res. 2014;57:165-175. doi:10.1016/j. jpsychires.2014.05.016

5. Xia K, Wang LF, Yang XC, et al. Comparing the effects of depression, anxiety, and comorbidity on quality-of-life, adverse outcomes, and medical expenditure in Chinese patients with acute coronary syndrome. Chin Med J. 2019;132(9):1045-1052. doi:10.1097/ CM9.0000000000000215
6. Zhang L, Zhao BJ, Yuan JR, Wang CF, Feng L, Jia XB. Comparison of chemical compositions in moutan cortex, paeoniae rubra radix and paeoniae alba radix based on "component structure" theory. J Chin Mater Med. 2016;41(10):1835-1842.

7. Li XL, Wu L, Wu WG, Yang HS. Effects of combined administration of paeoniae radix alba on local pharmacokinetics of six aconite alkaloids by skin microdialysis in vivo. $J$ Chin Mater Med. 2016;41(5):948-954.

8. Xie YF, Feng WW, Liu MC, et al. Investigation of efficacy enhancing and toxicity reducing mechanism of combination of aconiti lateralis radix praeparata and paeoniae radix alba in adjuvant-induced arthritis rats by metabolomics. Evid Based Complement Alternat Med. 2019;2019:9864841. doi:10.1155/2019/9864841

9. Jo GH, Kim SN, Kim MJ, Heo Y. Protective effect of paeoniae radix alba root extract on immune alterations in mice with atopic dermatitis. J Toxicol Environ Health A. 2018;81(12):502-511. doi:10.1080/15287394.2018.1460785

10. Wang Y, Gao SM, Li R, Zhang M, Gao S, Yu CQ. Antidepressant-like effects of the radix bupleuri and radix paeoniae alba drug pair. Neurosci Lett. 2016;633:14-20. doi:10.1016/j. neulet.2016.09.001

11. Wang YL, Wang JX, Hu XX, et al. Antidepressant-like effects of albiflorin extracted from radix paeoniae alba. J Ethnopharmacol. 2016;179:9-15. doi:10.1016/j.jep.2015.12.029

12. Ye XW, Deng YL, Xia LT, Ren HM, Zhang JL. Uncovering the mechanism of the effects of paeoniae radix alba on iron-deficiency anaemia through a network pharmacology-based strategy. BMC Complement Med Ther. 2020;20(1):130. doi:10.1186/s12906-020-02925-4

13. Huang KS, Lin JG, Lee HC, et al. Paeoniae alba radix promotes peripheral nerve regeneration. Evid Based Complement Alternat Med. 2011;2011:109809.

14. Zhang T, Lo CY, Xiao M, Cheng L, Pun Mok CK, Shaw PC. Antiinfluenza virus phytochemicals from radix paeoniae alba and characterization of their neuraminidase inhibitory activities. J Ethnopharmacol. 2020;253:112671. doi:10.1016/j.jep.2020.112671

15. Kong Y, Peng Q, Lv N, et al. Paeoniflorin exerts neuroprotective effects in a transgenic mouse model of Alzheimer's disease via activation of adenosine A(1) receptor. Neurosci Lett. 2020;730:135016. doi:10.1016/j.neulet.2020.135016

16. Liu M, Feng J, Du Q, Ai J, Lv Z. Paeoniflorin attenuates myocardial fibrosis in isoprenaline-induced chronic heart failure rats via inhibiting P38 MAPK pathway. Curr Med Sci. 2020;40 (2):307-312. doi:10.1007/s11596-020-2178-0

17. Huang X, Ren L, Hou L, et al. Paeoniflorin ameliorates antipsychotic-induced hyperprolactinemia in rats by attenuating impairment of the dopamine D2 receptor and TGF- $\beta 1$ signaling pathways in the hypothalamus and pituitary. $J$ Ethnopharmacol. 2020;257:112862. doi:10.1016/j.jep.2020.112862

18. Wang T, Xu L, Gao L, et al. Paeoniflorin attenuates early brain injury through reducing oxidative stress and neuronal apoptosis after subarachnoid hemorrhage in rats. Metab Brain Dis. 2020;35 (6):959-970. doi:10.1007/s11011-020-00571-w

19. She Y, Shao L, Zhang Y, et al. Neuroprotective effect of glycosides in buyang huanwu decoction on pyroptosis following cerebral ischemia-reperfusion injury in rats. $J$ Ethnopharmacol. 2019;242:112051. doi:10.1016/j.jep.2019.112051

20. Chen Y, Zhang R, Zhao W, et al. Paeoniflorin exhibits antitumor effects in nasopharyngeal carcinoma cells through downregulation of NEDD4. Am J Transl Res. 2019;11(12):7579-7590.

21. Wu W, Qiu C, Feng X, et al. Protective effect of paeoniflorin on acute cerebral infarction in rats. Curr Pharm Biotechnol. 2019.

22. Zhang L, Wei W. Anti-inflammatory and immunoregulatory effects of paeoniflorin and total glucosides of paeony. Pharmacol Ther. 2020;207:107452. doi:10.1016/j. pharmthera.2019.107452 
23. Chen LB, Qiu FM, Zhong XM, Hong C, Huang Z. Promoting neurogenesis in hippocampal dentate gyrus of chronic unpredictable stress-induced depressive-like rats with paeoniflorin. J Integr Neurosci. 2019;18(1):43-49.

24. Feng Y, Gao X, Meng M, Xue H, Qin X. Multi-omics reveals the mechanisms of antidepressant-like effects of the low polarity fraction of bupleuri radix. J Ethnopharmacol. 2020;256:112806. doi:10.1016/j.jep.2020.112806

25. Wang Y, Zhao L, Han X, et al. Saikosaponin a inhibits triple-negative breast cancer growth and metastasis through downregulation of CXCR4. Front Oncol. 2019;9:1487. doi: $10.3389 /$ fonc. 2019.01487

26. Pan $\mathrm{Y}, \mathrm{Ke} \mathrm{Z}$, Ye H, et al. Saikosaponin C exerts anti-HBV effects by attenuating HNF $1 \alpha$ and HNF $4 \alpha$ expression to suppress HBV pgRNA synthesis. Inflamm Res. 2019;68(12):1025-1034. doi:10.1007/s00011-019-01284-2

27. Jia $\mathrm{R}, \mathrm{Gu} \mathrm{Z}, \mathrm{He} \mathrm{Q}$, et al. Anti-oxidative, anti-inflammatory and hepatoprotective effects of radix bupleuri extract against oxidative damage in tilapia (oreochromis niloticus) via Nrf2 and TLRs signaling pathway. Fish Shellfish Immunol. 2019;93:395-405. doi:10.1016/j.fsi.2019.07.080

28. Yang F, Dong X, Yin X, Wang W, You L, Ni J. Radix bupleuri: a review of traditional uses, botany, phytochemistry, pharmacology, and toxicology. Biomed Res Int. 2017;2017:7597596.

29. Zeng LF, Cao Y, Wang L, et al. Role of medicinal plants for liver-qi regulation adjuvant therapy in post-stroke depression: a systematic review of literature. Phytother Res. 2017;31 (1):40-52. doi:10.1002/ptr.5740

30. Ye M, Bi YF, Ding L, Zhu WW, Gao W. Saikosaponin a functions as anti-epileptic effect in pentylenetetrazol induced rats through inhibiting mTOR signaling pathway. Biomed Pharmacother. 2016;81:281-287. doi:10.1016/j.biopha.2016.04.012

31. Lee TH, Park S, You MH, Lim JH, Min SH, Kim BM. A potential therapeutic effect of saikosaponin $\mathrm{C}$ as a novel dual-target anti-Alzheimer agent. $J$ Neurochem. 2016;136(6):1232-1245. doi: $10.1111 /$ jnc. 13515

32. Fu R, Zhang L, Li Y, et al. Saikosaponin D inhibits autophagosome-lysosome fusion and induces autophagy-independent apoptosis in MDA-MB-231 breast cancer cells. Mol Med Rep. 2020;22 (2):1026-1034. doi:10.3892/mmr.2020.11155

33. Mani JS, Johnson JB, Steel JC, et al. Natural product-derived phytochemicals as potential agents against coronaviruses: a review. Virus Res. 2020;284:197989. doi:10.1016/j.virusres.2020.197989

34. Feng P, Xu Y, Tong B, et al. Saikosaponin a attenuates hyperlipidemic pancreatitis in rats via the PPAR- $\gamma / \mathrm{NF}-\mathrm{\kappa B}$ signaling pathway. Exp Ther Med. 2020;19(2):1203-1212.

35. Su J, Pan YW, Wang SQ, Li XZ, Huang F, Ma SP. Saikosaponin$\mathrm{d}$ attenuated lipopolysaccharide-induced depressive-like behaviors via inhibiting microglia activation and neuroinflammation. Int Immunopharmacol. 2020;80:106181. doi:10.1016/j. intimp.2019.106181

36. Liu L, Yan J, Ge F, et al. Saikosaponin-D improves fear memory deficits in ovariectomized rats via the action of estrogen receptor- $\alpha$ in the hippocampus. Mol Med Rep. 2019;20(1):332-340.

37. Hong Y, Deng N, Jin HN, et al. Saikosaponin A modulates remodeling of Kv4.2-mediated A-type voltage-gated potassium currents in rat chronic temporal lobe epilepsy. Drug Des Devel Ther. 2018;12:2945-2958. doi:10.2147/DDDT.S166408

38. Shin JS, Im HT, Lee KT. Saikosaponin B2 suppresses inflammatory responses through $\mathrm{IKK} / \mathrm{I} \kappa \mathrm{B} \alpha / \mathrm{NF}-\mathrm{\kappa B}$ signaling inactivation in LPS-induced raw 264.7 macrophages. Inflammation. 2019;42 (1):342-353. doi:10.1007/s10753-018-0898-0

39. Zhang Y, Gu L, Xia Q, Tian L, Qi J, Cao M. Radix astragali and radix angelicae sinensis in the treatment of idiopathic pulmonary fibrosis: a systematic review and meta-analysis. Front Pharmacol. 2020;11:415. doi:10.3389/fphar.2020.00415
40. Gong W, Zhu S, Chen C, et al. The anti-depression effect of angelicae sinensis radix is related to the pharmacological activity of modulating the hematological anomalies. Front Pharmacol. 2019;10:192.

41. Song L, Zhou QH, Wang HL, et al. Chinese herbal medicine adjunct therapy in patients with acute relapse of multiple sclerosis: a systematic review and meta-analysis. Complement Ther Med. 2017;31:71-81. doi:10.1016/j.ctim.2017.02.004

42. Xu YJ, Mei Y, Qu ZL, et al. Ligustilide ameliorates memory deficiency in APP/PS1 transgenic mice via restoring mitochondrial dysfunction. Biomed Res Int. 2018;2018:4606752. doi: $10.1155 / 2018 / 4606752$

43. Lu Y, Hsiang F, Chang JH, et al. Houshiheisan and its components promote axon regeneration after ischemic brain injury. Neural Regen Res. 2018;13(7):1195-1203. doi:10.4103/1673-5374.235031

44. Zhong LJ, Hua YL, Ji P, et al. Evaluation of the anti-inflammatory effects of volatile oils from processed products of angelica sinensis radix by GC-MS-based metabolomics. $J$ Ethnopharmacol. 2016;191:195-205.

45. Gong W, Zhou Y, Li X, et al. Neuroprotective and cytotoxic phthalides from angelicae sinensis radix. Molecules. 2016;21 (5):549. doi:10.3390/molecules21050549

46. Deng M, Sun H, Shen J, Fan Y, Zhang L, Zhang J. Radix angelica sinensis promotes synaptic plasticity during cognitive recovery in chronically stressed rats. Curr Neurovasc Res. 2015;12 (3):232-239. doi:10.2174/1567202612666150603125710

47. Zhou QM, Zhang JJ, Li S, Chen S. n-butylidenephthalide treatment prolongs life span and attenuates motor neuron loss in SOD1(G93A) mouse model of amyotrophic lateral sclerosis. CNS Neurosci Ther. 2017;23(5):375-385. doi:10.1111/cns.12681

48. Harn HJ, Lin SZ, Lin PC, et al. Local interstitial delivery of z-butylidenephthalide by polymer wafers against malignant human gliomas. Neuro-Oncology. 2011;13(6):635-648. doi:10.1093/neuonc/ nor021

49. Zhao YH, Liu NW, Ke CC, et al. Combined treatment of sodium ferulate, n-butylidenephthalide, and ADSCs rehabilitates neurovascular unit in rats after photothrombotic stroke. J Cell Mol Med. 2019;23(1):126-142. doi:10.1111/jcmm.13894

50. Chi K, Fu RH, Huang YC, et al. Adipose-derived stem cells stimulated with n-butylidenephthalide exhibit therapeutic effects in a mouse model of parkinson's disease. Cell Transplant. 2018;27(3):456-470. doi:10.1177/0963689718757408

51. Rajamani K, Liu JW, Wu CH, et al. n-Butylidenephthalide exhibits protection against neurotoxicity through regulation of tryptophan 2, 3 dioxygenase in spinocerebellar ataxia type 3 . Neuropharmacology. 2017;117:434-446. doi:10.1016/j. neuropharm.2017.02.014

52. Chang CY, Chen SM, Lu HE, et al. N-butylidenephthalide attenuates alzheimer's disease-like cytopathy in down syndrome induced pluripotent stem cell-derived neurons. Sci Rep. 2015;5 (1):8744. doi:10.1038/srep08744

53. Nam KN, Kim KP, Cho KH, et al. Prevention of inflammation-mediated neurotoxicity by butylidenephthalide and its role in microglial activation. Cell Biochem Funct. 2013;31 (8):707-712. doi:10.1002/cbf.2959

54. Yuan HL, Zhao YL, Ding CF, et al. Anti-inflammatory and antinociceptive effects of Curcuma kwangsiensis and its bioactive terpenoids in vivo and in vitro. $J$ Ethnopharmacol. 2020;259:112935. doi:10.1016/j.jep.2020.112935

55. Wang Q, Yuan LL, Zhang YL, Fan WT. Research on network pharmacology of acori tatarinowii rhizoma combined with curcumae radix in treating epilepsy. Zhongguo Zhong Yao Za Zhi. 2019;44(13):2701-2708.

56. Kaya P, Lee SR, Lee YH, et al. Curcumae radix extract decreases mammary tumor-derived lung metastasis via suppression of C-C chemokine receptor type 7 expression. Nutrients. 2019;11 (2):410. doi:10.3390/nu11020410 
57. Fan WT, Wang Q. Mechanism of acori tatarinowii rhizoma-curcumae radix treating depression based on network pharmacology. Zhongguo Zhong Yao Za Zhi. 2018;43(12):2607-2611.

58. Zhang J, Song Y, Wang QH, et al. Effects of curcumae rhizoma/ vinegar-processed curcumae rhizoma on immune hepatic fibrosis, proliferation of HSC-T6 and expression of $\alpha$-SMA and procollagen I. J Chin Mater Med. 2017;42(13):2538-2545.

59. Fu WJ, Lei T, Yin Z, et al. Anti-atherosclerosis and cardio-protective effects of the angong niuhuang pill on a high fat and vitamin D3 induced rodent model of atherosclerosis. $J$ Ethnopharmacol. 2017;195:118-126. doi:10.1016/j.jep.2016.11.015

60. Wang ZY, Liu JG, Li H, Yang HM. Pharmacological effects of active components of chinese herbal medicine in the treatment of alzheimer's disease: a review. Am J Chin Med. 2016;44 (8):1525-1541. doi:10.1142/S0192415X16500853

61. Katasonov AB. Curcumin as an adjuvant treatment of depression: mechanisms of action and application prospects. Zh Nevrol Psikhiatr Im $\quad S \quad S$ Korsakova. 2020;120(2):125-131. doi:10.17116/jnevro2020120021125

62. Asadi S, Gholami MS, Siassi F, Qorbani M, Sotoudeh G. Beneficial effects of nano-curcumin supplement on depression and anxiety in diabetic patients with peripheral neuropathy: a randomized, double-blind, placebo-controlled clinical trial. Phytother Res. 2020;34(4):896-903. doi:10.1002/ptr.6571

63. Naqvi F, Saleem S, Naqvi F, et al. Curcumin lessens unpredictable chronic mild stress-induced depression and memory deficits by modulating oxidative stress and cholinergic activity. Pak J Pharm Sci. 2019;32(4(Supplementary)):1893-1900.

64. Bhat A, Mahalakshmi AM, Ray B, et al. Benefits of curcumin in brain disorders. BioFactors. 2019;45(5):666-689. doi:10.1002/biof.1533

65. Chen DQ, Wang YN, Vaziri ND, Chen L, Hu HH, Zhao YY. Poricoic acid A activates AMPK to attenuate fibroblast activation and abnormal extracellular matrix remodelling in renal fibrosis, phytomedicine: international. $J$ Phytother Phytopharmacol. 2020;72:153232. doi:10.1016/j.phymed.2020.153232

66. Hwang YH, Jang SA, Lee A, Kim T, Ha H. Poria cocos ameliorates bone loss in ovariectomized mice and inhibits osteoclastogenesis in vitro. Nutrients. 2020;12(5):1383. doi:10.3390/nu12051383

67. Jiang Y, Fan L. Evaluation of anticancer activities of Poria cocos ethanol extract in breast cancer: in vivo and in vitro, identification and mechanism. $J$ Ethnopharmacol. 2020;257:112851. doi:10.1016/j.jep.2020.112851

68. Pang Y, Zhu S, Pei H. Pachymic acid protects against cerebral ischemia/reperfusion injury by the PI3K/Akt signaling pathway. Metab Brain Dis. 2020;35(4):673-680. doi:10.1007/s11011-02000540-3

69. Chen C, Yin Q, Tian J, et al. Studies on the potential link between antidepressant effect of Xiaoyao San and its pharmacological activity of hepatoprotection based on multi-platform metabolomics. J Ethnopharmacol. 2020;249:112432. doi:10.1016/j.jep.2019.112432

70. Kim JH, Sim HA, Jung DY, et al. Poria cocus wolf extract ameliorates hepatic steatosis through regulation of lipid metabolism, inhibition of ER stress, and activation of autophagy via AMPK activation. Int J Mol Sci. 2019;20(19).

71. Wang H, Shi S, Wang S. Can highly cited herbs in ancient traditional Chinese medicine formulas and modern publications predict therapeutic targets for diabetes mellitus? J Ethnopharmacol. 2018;213:101-110. doi:10.1016/j.jep.2017.10.032

72. Yu M, Xu X, Jiang N, et al. Dehydropachymic acid decreases bafilomycin A1 induced $\beta$-Amyloid accumulation in PC12 cells. J Ethnopharmacol. 2017;198:167-173. doi:10.1016/j. jep.2017.01.007

73. Kamalakannan Preethi O, Sampath V, Ravikumar N, Mahalaxmi S. Comparative evaluation of physicochemical properties and apical sealing ability of a resin sealer modified with pachymic acid. Eur Endod J. 2020;5(1):23-27.
74. Liu Z, Qin G, Mana L, et al. GAPT regulates cholinergic dysfunction and oxidative stress in the brains of learning and memory impairment mice induced by scopolamine. Brain Behav. 2020;10(5):e01602. doi:10.1002/brb3.1602

75. Zhang CY, Zhu JY, Ye Y, et al. Erhuang formula ameliorates renal damage in adenine-induced chronic renal failure rats via inhibiting inflammatory and fibrotic responses. Biomed Pharmacother. 2017;95:520-528. doi:10.1016/j. biopha.2017.08.115

76. Li JY, Wu HX, Yang G. Pachymic acid improves survival and attenuates acute lung injury in septic rats induced by cecal ligation and puncture. Eur Rev Med Pharmacol Sci. 2017;21 (8):1904-1910.

77. Shah VK, Choi JJ, Han JY, Lee MK, Hong JT, Oh KW. Pachymic acid enhances pentobarbital-induced sleeping behaviors via GABAA-ergic systems in mice. Biomol Ther (Seoul). 2014;22 (4):314-320. doi:10.4062/biomolther.2014.045

78. Lee $\mathrm{YH}$, Lee NH, Bhattarai G, et al. Anti-inflammatory effect of pachymic acid promotes odontoblastic differentiation via HO-1 in dental pulp cells. Oral Dis. 2013;19(2):193-199. doi:10.1111/ j.1601-0825.2012.01970.x

79. Li TH, Hou CC, Chang CL, Yang WC. Anti-hyperglycemic properties of crude extract and triterpenes from Poria cocos. Evid Based Complement Alternat Med. 2011;2011:1-8. doi:10.1155/2011/128402

80. Zhu KY, Fu Q, Xie HQ, et al. Quality assessment of a formulated Chinese herbal decoction, Kaixinsan, by using rapid resolution liquid chromatography coupled with mass spectrometry: a chemical evaluation of different historical formulae. J Sep Sci. 2010;33(23-24):3666-3674. doi:10.1002/ jssc. 201000498

81. Lu J, Fu L, Qin G, Shi P, Fu W. The regulatory effect of xiaoyao san on glucocorticoid receptors under the condition of chronic stress. Cell Mol Biol. 2018;64(6):103-109. doi:10.14715/cmb/ 2018.64.6.17

82. Chen Y, Wang X, Zhu Y, et al. Synthesis of a hexavalent betulinic acid derivative as a hemagglutinin-targeted influenza virus entry inhibitor. Mol Pharm. 2020;17(7):2546-2554. doi:10.1021/acs. molpharmaceut.0c00244

83. Lo WL, Hsu TI, Yang WB, et al. Betulinic acid-mediated tuning of PERK/CHOP signaling by Sp1 inhibition as a novel therapeutic strategy for glioblastoma. Cancers. 2020;12(4). doi:10.3390/ cancers 12040981

84. Deng G, Ma C, Zhao H, et al. Anti-edema and antioxidant combination therapy for ischemic stroke via glyburide-loaded betulinic acid nanoparticles. Theranostics. 2019;9 (23):6991-7002. doi:10.7150/thno.35791

85. Ghosh A, Tiwari GJ, Panda CK. Role of radical quenching activity of dihydrocanaric acid in the treatment of cancer-experimental and theoretical. Biotech. 2020;10(6):251.

86. Cao S, Han Y, Li Q, et al. Mapping pharmacological network of multi-targeting litchi ingredients in cancer therapeutics. Front Pharmacol. 2020;11:451.

87. Navabi SP, Sarkaki A, Mansouri E, Badavi M, Ghadiri A, Farbood Y. The effects of betulinic acid on neurobehavioral activity, electrophysiology and histological changes in an animal model of the alzheimer's disease. Behav Brain Res. 2018;337:99-106. doi:10.1016/j.bbr.2017.10.002

88. Machado DG, Cunha MP, Neis VB, et al. Antidepressant-like effects of fractions, essential oil, carnosol and betulinic acid isolated from rosmarinus officinalis L. Food Chem. 2013;136 (2):999-1005. doi:10.1016/j.foodchem.2012.09.028

89. Shen ZM, Zhu ML, Zhao AQ. Comparative observation on efficacy of jieyu pill and maprotiline in treating depression. Zhongguo Zhong Xi Yi Jie He Za Zhi Zhongguo Zhongxiyi Jiehe Zazhi. 2004;24(5):415-417. 
90. Liu J, Jia HX, Wang JQ, Xu Y, Tian JZ, Shi J. Treatment of refractory depression patients of yin deficiency inner heat syndrome by jieyu granule combined paroxetine: an efficacy observation. Chin J Integr Med. 2013;33(4):462-465.

91. Yu Y, Zhang G, Han T, Li J, Huang HL. Network meta-analysis of Chinese patent medicines in treatment of liver stagnation and spleen deficiency of depression. Zhongguo Zhong Yao Za Zhi. 2019;44(23):5217-5224.

92. Feng BL, Wang QC, Li ZY. Influence of jieyu huoxue decoction on rehabilitation of patients with depression after cerebral infarction. Zhong Xi Yi Jie He Xue Bao. 2004;2(3):182-184. doi:10.3736/jcim20040309

93. Wang $\mathrm{C}$, Wu C, Yan Z, Cheng X. Ameliorative effect of Xiaoyao-jieyu-san on post-stroke depression and its potential mechanisms. J Nat Med. 2019;73(1):76-84. doi:10.1007/s11418018-1243-5

94. Mao QQ, Ip SP, Ko KM, Tsai SH, Che CT. Peony glycosides produce antidepressant-like action in mice exposed to chronic unpredictable mild stress: effects on hypothalamic-pituitaryadrenal function and brain-derived neurotrophic factor. Prog Neuropsychopharmacol Biol Psychiatry. 2009;33(7):1211-1216. doi:10.1016/j.pnpbp.2009.07.002

95. Zhong X, Li G, Qiu F, Huang Z. Paeoniflorin ameliorates chronic stress-induced depression-like behaviors and neuronal damages in rats via activation of the ERK-CREB pathway. Front Psychiatry. 2018;9:772. doi:10.3389/fpsyt.2018.00772

96. Yu JB, Zhao ZX, Peng R, et al. Gut microbiota-based pharmacokinetics and the antidepressant mechanism of paeoniflorin. Front Pharmacol. 2019;10:268. doi:10.3389/fphar.2019.00268
97. Chao B, Huang S, Pan J, Zhang Y, Wang Y. Saikosaponin d downregulates microRNA-155 and upregulates FGF2 to improve depression-like behaviors in rats induced by unpredictable chronic

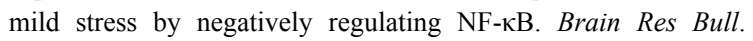
2020;157:69-76. doi:10.1016/j.brainresbull.2020.01.008

98. Yang M, Dang R, Xu P, et al. Dl-3-n-butylphthalide improves lipopolysaccharide-induced depressive-like behavior in rats:

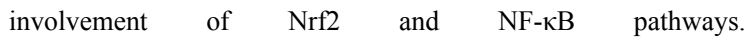
Psychopharmacology. 2018;235(9):2573-2585. doi:10.1007/ s00213-018-4949-x

99. Wang Z, Ren W, Zhao F, Han Y, Liu C, Jia K. Curcumin amends $\mathrm{Ca} 2+$ dysregulation in microglia by suppressing the activation of P2X7 receptor. Mol Cell Biochem. 2020;465(1-2):65-73. doi:10.1007/s11010-019-03668-8

100. Fan C, Song Q, Wang P, Li Y, Yang M, Yu SY. Neuroprotective effects of curcumin on IL-1 $\beta$-induced neuronal apoptosis and depression-like behaviors caused by chronic stress in rats. Front Cell Neurosci. 2018;12:516. doi:10.3389/fncel.2018.00516

101. Zhang WY, Guo YJ, Han WX, et al. Curcumin relieves depressive-like behaviors via inhibition of the NLRP3 inflammasome and kynurenine pathway in rats suffering from chronic unpredictable mild stress. Int Immunopharmacol. 2019;67:138-144. doi:10.1016/j.intimp.2018.12.012

102. Zhou Y, Zhou B, Pache L, et al. Metascape provides a biologist-oriented resource for the analysis of systems-level datasets. Nat Commun. 2019;10(1):1523. doi:10.1038/s41467019-09234-6
Neuropsychiatric Disease and Treatment

\section{Publish your work in this journal}

Neuropsychiatric Disease and Treatment is an international, peerreviewed journal of clinical therapeutics and pharmacology focusing on concise rapid reporting of clinical or pre-clinical studies on a range of neuropsychiatric and neurological disorders. This journal is indexed on PubMed Central, the 'PsycINFO' database and CAS, and
Dovepress

is the official journal of The International Neuropsychiatric Association (INA). The manuscript management system is completely online and includes a very quick and fair peer-review system, which is all easy to use. Visit http://www.dovepress.com/testimonials.php to read real quotes from published authors. 